\title{
Growth of zinc-blende GaN on muscovite mica by molecular beam epitaxy
}

\author{
Bruno Daudin ${ }^{1}$, Fabrice Donatini ${ }^{2}$, Catherine Bougerol ${ }^{2}$, Bruno Gayral ${ }^{1}$, Edith Bellet- \\ Amalric $^{1}$, Rémy Vermeersch ${ }^{1}$, Nathaniel Feldberg ${ }^{1}$, Jean-Luc Rouvière ${ }^{3}$, Maria José Recio \\ Carretero $^{4}$, Núria Garro ${ }^{4}$, Saül Garcia-Orrit ${ }^{4}$ and Ana $\mathrm{Cros}^{4}$ \\ ${ }^{1}$ Univ. Grenoble-Alpes, CEA-IRIG, PHELIQS, 17 av. des Martyrs, 38000 Grenoble, France. \\ ${ }^{2}$ Univ. Grenoble-Alpes, CNRS-Institut Néel, 25 av. des Martyrs, 38000 Grenoble, France. \\ ${ }^{3}$ Univ. Grenoble-Alpes, CEA-IRIG, MEM, 17 av. des Martyrs, 38000 Grenoble, France. \\ ${ }^{4}$ Institute of Materials Science (ICMUV), Universidad de Valencia, P.O. Box 22085, Valencia, \\ Spain
}

Corresponding author: bruno.daudin@cea.fr

\begin{abstract}
The mechanisms of plasma-assisted molecular beam epitaxial growth of GaN on muscovite mica were investigated. Using a battery of techniques, including scanning and transmission electron microscopy, atomic force microscopy, cathodoluminescence, Raman spectroscopy and X-ray diffraction, it was possible to establish that, in spite of the lattice symmetry mismatch, GaN grows in epitaxial relationship with mica, with the [11-20] GaN direction parallel to [010] direction of mica. GaN layers could be easily detached from the substrate via the delamination of the upper layers of the mica itself, discarding the hypothesis of a van der Waals growth mode. Mixture of wurtzite (hexagonal) and zinc blende (cubic) crystallographic phases was found in the GaN layers with ratios highly dependent on the growth conditions. Interestingly, almost pure zinc blende $\mathrm{GaN}$ epitaxial layers could be obtained at high growth temperature, suggesting the existence of a specific GaN nucleation mechanism on mica and opening a new way to the growth of the thermodynamically less stable zinc blende GaN phase.
\end{abstract}




\section{Introduction}

The lack of cheap and widely available lattice-adapted substrates is still limiting the potentialities of the III-nitride semiconductor family ( $\mathrm{GaN}$, AlN InN and their alloys) for optoelectronic and electronic applications, despite a continuous progress along the past years. In particular, the development of specific growth strategies has succeeded in the reduction of the density of extended defects in epitaxial layers of GaN grown on sapphire to a level acceptable for the realization of optoelectronic devices. Similarly, the quality of GaN grown on $\mathrm{Si}$ is continuously progressing in spite of the lattice and thermal mismatch between both materials. These extended defect reduction strategies are, nevertheless, costly, which motivates the long-lasting quest for alternative substrates. This includes the current interest in van der Waals (VdW) epitaxy, which relies on the prospect to grow mismatched layers with a reduced amount of strain by circumventing the lattice matching requirement in absence of strong covalent bonding to the substrate surface[1]. This field started with the pioneering work of Ueno on the heteroepitaxy of $\mathrm{MoSe}_{2}$ and $\mathrm{NbSe}_{2}$ on muscovite mica[2]. More recently, the growth of transition metal dichalcogenides on graphene and the successful realization of monolayer-thick electronic devices have been reported, as an illustration of the potential of VdW epitaxy technique[3].

This approach, recently extended to the III-nitride semiconductor family[4-7], has not met the initial expectations due to the lack of strong bonds at the epilayer/graphene interface and to the poor wetting of graphene by Ga. Therefore, the growth of continuous layers is still challenging[7]. It was established that during the first stages of the growth of GaN layers on graphene/SiC the $\mathrm{GaN}$ seed orientation was governed by graphene-GaN lattice coincidences rather than by the structure of the subjacent $\mathrm{SiC}$ material. Accordingly, besides seeds with the standard <0001> wurtzite (WZ) orientation, several other epitaxial relationships were observed corresponding to m-plane and a-plane oriented seeds as well as to seeds exhibiting other possible lattice coincidence relationships. This eventually led to the formation of a discontinuous layer of non-coalesced grains with a significant fraction of zinc blende (ZB) $\mathrm{GaN}$ during the nucleation stage and the presence of stacking faults[8]. More fundamentally, it was theoretically established that the growth mode on graphene, instead of Volmer-Weber or Frank-van der Merwe type, was critically depending on the presence of defects modulating the adherence between the graphene and the epitaxial layer[9]. 
In this article, we focus on the growth of $\mathrm{GaN}$ on muscovite mica, a layered material available in large quantities and at low price. Mica consists of sheets weakly bound by electrostatic interactions with $\mathrm{K}$ atoms, making it a potential VdW substrate for GaN growth: as a matter of fact, the successful growth of (000-1) oriented WZ GaN by pulsed laser deposition was reported by Matsuki and co-workers[10]. As concerns plasma assisted molecular beam epitaxy (PA-MBE) growth technique used in the present work, fully coalesced GaN layers in single epitaxial relationship to mica will be demonstrated, indicating some degree of chemical bonding to the mica surface layer formed of $\mathrm{Si} / \mathrm{Al}$ atoms tetrahedrally coordinated to O. Remarkably, depending on growth parameters, GaN layers predominantly exhibiting the ZB structure are obtained. This crystallographic GaN variant, thermodynamically less stable than the $\mathrm{WZ}$ one, is of potential interest for opto-electronic applications. The growth of $\mathrm{ZB} \mathrm{GaN}$ was reported on GaAs $(001)[11,12]$, free-standing cubic 3C-SiC (001)[13] or SiC/Si (001)[14,15] pseudo substrates elaborated by chemical vapour deposition. However, surface roughness promoting the formation of (111)-oriented facets was detrimental to the ZB phase purity. Accordingly, Ga-rich conditions favouring layer smoothness were found to be optimal for ZB GaN growth[16]. Although the growth of ZB $\mathrm{GaN}$ on $\mathrm{SiC}$ (0001) was successfully reported by using $\mathrm{Mn}$ as a dopant to trigger $\mathrm{ZB}$ phase nucleation[17], substrates with six-fold or three-fold symmetry such as SiC (0001) or Si (111) tend to favour the growth of the most stable WZ phase. In such a case, decreasing the growth temperature and concomitantly the mobility of atomic species on the surface allows the formation of $\mathrm{ZB}$ material. The counterpart, however, is the favoured incorporation of impurities at low temperature as well as the formation of stacking faults, detrimental to structural and optical properties. In this context, the remarkable morphology of atomically flat muscovite mica substrates was found to lead, under proper growth conditions, to nucleation of ZB GaN in spite of the crystallographic symmetry mismatch, opening a new route for the growth of this $\mathrm{GaN}$ variant.

\section{Results and discussion}

Freshly cleaved commercial muscovite crystal plates were used as substrates. They were fixed with In on the sample holder. After introduction in the molecular beam epitaxy (MBE) growth chamber and annealing during 30 minutes at $250{ }^{\circ} \mathrm{C}$ in order to eliminate adsorbed species on the surface, the substrates were heated up to the growth temperature, which ranged from 700 to $860^{\circ} \mathrm{C}$. The temperature was measured using a thermocouple facing the rear side of the sample holder. The growth conditions of the different samples are given in 
table 1. The $\Phi_{\mathrm{Ga}} / \Phi_{\mathrm{N}}$ ratio value refers to nominal atomic flux values, irrespective of temperature-dependent $\mathrm{Ga}$ desorption rate. However, the observation of $\mathrm{Ga}$ droplets on the surface was an a posteriori confirmation of Ga-rich growth conditions for samples D and D'.

\begin{tabular}{|c|c|c|c|c|c|}
\hline Sample & A & B & C & D & D' \\
\hline $\mathrm{T}_{\mathrm{G}}\left({ }^{\circ} \mathrm{C}\right)$ & 700 & 800 & 850 & 850 & 860 \\
\hline Nominal $\Phi_{\mathrm{Ga}} / \Phi_{\mathrm{N}}$ & $<1$ & $<1$ & $<1$ & $>1$ & $>1$ \\
\hline
\end{tabular}

Table 1: List of samples studied and growth conditions

The morphology of the films was investigated by optical and scanning electron microscopy. It was found that layers were continuous and consisted of closely packed grains exhibiting different morphologies. As shown in figure 1a for sample B, macroscopic features exhibiting "phone cord buckle" morphology were also observed for all samples. Such buckles are assigned to the relaxation of the compressive strain experienced by a film poorly bound to the substrate[18-20], consistent at first sight with the VdW epitaxy mechanism expected for the deposition of GaN on mica. Further zooming (atomic force microscopy (AFM) images shown in figure 1) puts in evidence that the GaN layer exhibits the same granular morphology in the buckles and out of them (figure 1c and d).
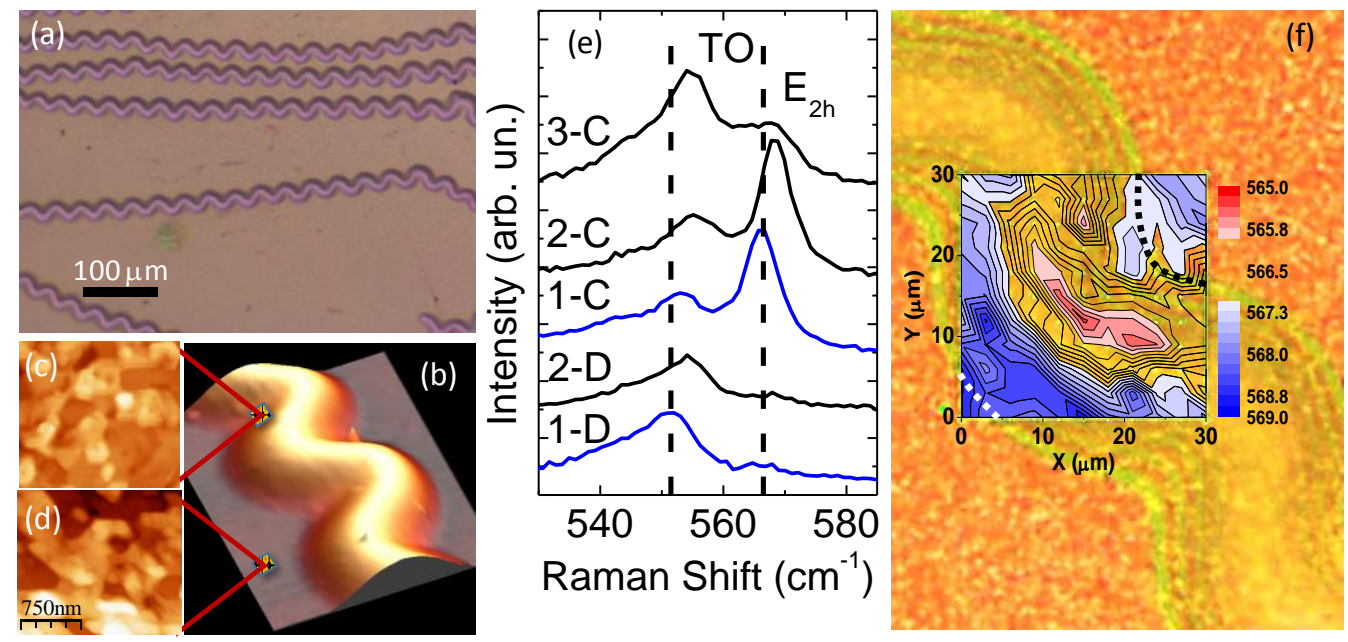

Figure 1: Morphology and strain of a GaN layer grown on mica. a) optical microscopy view graph showing a continuous layer with phone cord buckles on sample B. b) 
AFM image of a phone cord buckle in sample D. c) microstructure of the GaN layer on the buckle. d) The same out of the buckle. e) Comparison of the Raman spectra from different locations of samples $C$ and D. Locations 1 correspond to a buckle. The vertical lines mark the frequency of relaxed wurtzite $\left(E_{2 h}\right.$ mode at $\left.566.5 \mathrm{~cm}^{-1}\right)$ and zinc-blende (TO mode at 551.5 $\left.\mathrm{cm}^{-1}\right)$ GaN. The spectra were vertically shifted for clarity. f) Optical micrograph of a buckle from sample B. The superposed color map gives the peak frequency (in $\mathrm{cm}^{-1}$ ) of the $E_{2 h}$ phonon mode, as obtained from the Raman spectra at each point of the map $(30 \times 30 \mu m, 121$ points, $50 \times$ objective).

Raman spectroscopy experiments were performed with the purpose of determining the crystal quality, phase and strain state of $\mathrm{GaN}$ in different spots along the layer, in particular in the buckle region and out of it. Measurements were performed in backscattering configuration along the growth direction using the $532 \mathrm{~nm}$ line of a laser diode as excitation source. A Horiba Jobin Yvon Xplora spectrometer equipped with a Peltier-cooled charge coupled device detector and 2400 grooves/mm diffraction gratings was used for spectral analysis. A 100x microscope objective focused the excitation laser on the sample and collected the scattered light to the spectrometer. Figure 1e shows the Raman spectra of samples C and D taken at different locations, revealing crystallographic phase mixing and its dependence on growth conditions. The TO mode, characteristic of the ZB phase, is observed in all spectra, but with variable intensity and frequencies ranging from $551.5 \mathrm{~cm}^{-1}$ to $555.0 \mathrm{~cm}^{-1}$. The $\mathrm{E}_{2 \mathrm{~h}}$ mode, a signature of the $\mathrm{WZ}$ phase, can be observed in most of the samples, with frequencies varying from $566.3 \mathrm{~cm}^{-1}$ to $568.4 \mathrm{~cm}^{-1}$. However, in sample D this mode is very weak or absent, depending on the analyzed spot. In sample $\mathrm{C}$ the relative intensity of the ZB to WZ-phase peaks depends strongly on location. Spectra 1-C and 1-D are taken at the top part of the phone cord buckles. It is interesting to note that the relative intensity of the $\mathrm{WZ}$ and $\mathrm{ZB}$ modes in the buckles is usually the same as that of the surrounding material, from which the buckles originate. The main difference is a clear red-shift of the peaks towards their relaxed values, pointed out with vertical lines in the figure.

This is a straight-forward indication that the strain accumulated in the layers is almost completely relaxed through the formation of buckles. Based on the biaxial strain approximation, we can estimate the mean strain in the flat layers: $e_{x x}=-0.18 \%$ for sample $C$ and $e_{x x}=-0.17 \%$ for sample D. However, the strain distribution is quite inhomogeneous, 
especially around the buckles. Figure 1f shows the optical micrograph of a buckle from sample B. Superimposed to it we have plotted a color map showing the variation of the peak frequency of the $\mathrm{E}_{2 \mathrm{~h}}$ phonon mode, as obtained from the Raman spectra taken at each point of the map. The frequency of this mode is proportional to strain[21]. Keeping this in mind, the color scale has been chosen so that the relaxed regions are transparent, red indicates a shift of the peak to lower frequencies (tensile strain) and blue a shift to higher frequency (compressive strain). The top of the buckle is relaxed or under small tensile strain, while its convex flank is under small compressive strain. For all samples we have determined the strain by using the frequency of the $\mathrm{E}_{2 \mathrm{~h}}$ mode of the hexagonal phase and taking the value of $566.5 \mathrm{~cm}^{-1}$ as a relaxed reference. The value of the deformation potentials has been taken from Davidov et al[21].

The layers were further analyzed by X-ray diffraction (HRXRD) using a Rigaku SmartLab diffractometer. Out-of-plane $2 \theta^{-} \theta$ scans results, using a 2 reflection $\operatorname{Ge}(220)$ monochromator and a long plate collimator $\left(0.228^{\circ}\right)$, are shown in figure $2 \mathrm{a}$, confirming that in all cases the $\mathrm{GaN}$ layers consisted of a mixture of $\mathrm{ZB}$ and $\mathrm{WZ}$ phases. However, the ratio between both phases was markedly dependent on growth conditions. Remarkably, in the case of samples D and D', the ZB phase was predominant, with a very small amount of WZ phase. The crystalline quality of both the ZB and WZ grains was similar, assessed by the same width in the $2 \theta^{-} \theta$ scans and the same mosaicity ( $\omega$-scans). The prevalence of ZB phase in sample D' was further studied by transmission electron microscopy (TEM). The sample was prepared in cross section by mechanical polishing followed by ion milling using Gatan PIPS equipment. High Resolution TEM images were obtained on a FEI Tecnai microscope operated at $200 \mathrm{kV}$. The image (figure 2b) and the corresponding FFT given in inset are characteristic of cubic symmetry, viewed along the [0-1-1] zone axis. A few defects corresponding to microtwins are visible, as commonly observed in other semiconductors of cubic symmetry. No evidence of hexagonal stacking sequence was observed.

The two parameters conventionally controlling the stability of the $\mathrm{ZB}$ phase are the growth temperature and the substrate roughness: due to the thermodynamically less stable character of the $\mathrm{ZB}$ phase with respect to its $\mathrm{WZ}$ counterpart, the $\mathrm{ZB}$ phase is generally grown at relatively lower temperature than WZ [12]. More specifically, it was established that (111) nanofacets associated with substrate surface roughness were responsible for the nucleation of the more stable $\mathrm{WZ}$ phase, leading to an irreversible crystallographic phase mixing and 
increasing WZ fraction with layer thickness[16,22]. Then, N-rich MBE growth conditions, associated with surface roughening, are not favourable to the growth of pure ZB phase. Consistently with the above statement, samples D and D' exhibiting a negligible WZ fraction, were grown in Ga-rich conditions. However, the growth temperature was high, which would be expected to favour the formation of the WZ phase but this did not hamper ZB growth. Although the details of the nucleation process at the atomic scale are still unclear, these features suggest that the remarkable smoothness of mica and also possibly its surface chemistry are favourable to the nucleation of $\mathrm{ZB} \mathrm{GaN}$ as well as to maintain $\mathrm{ZB}$ phase purity once initiated.

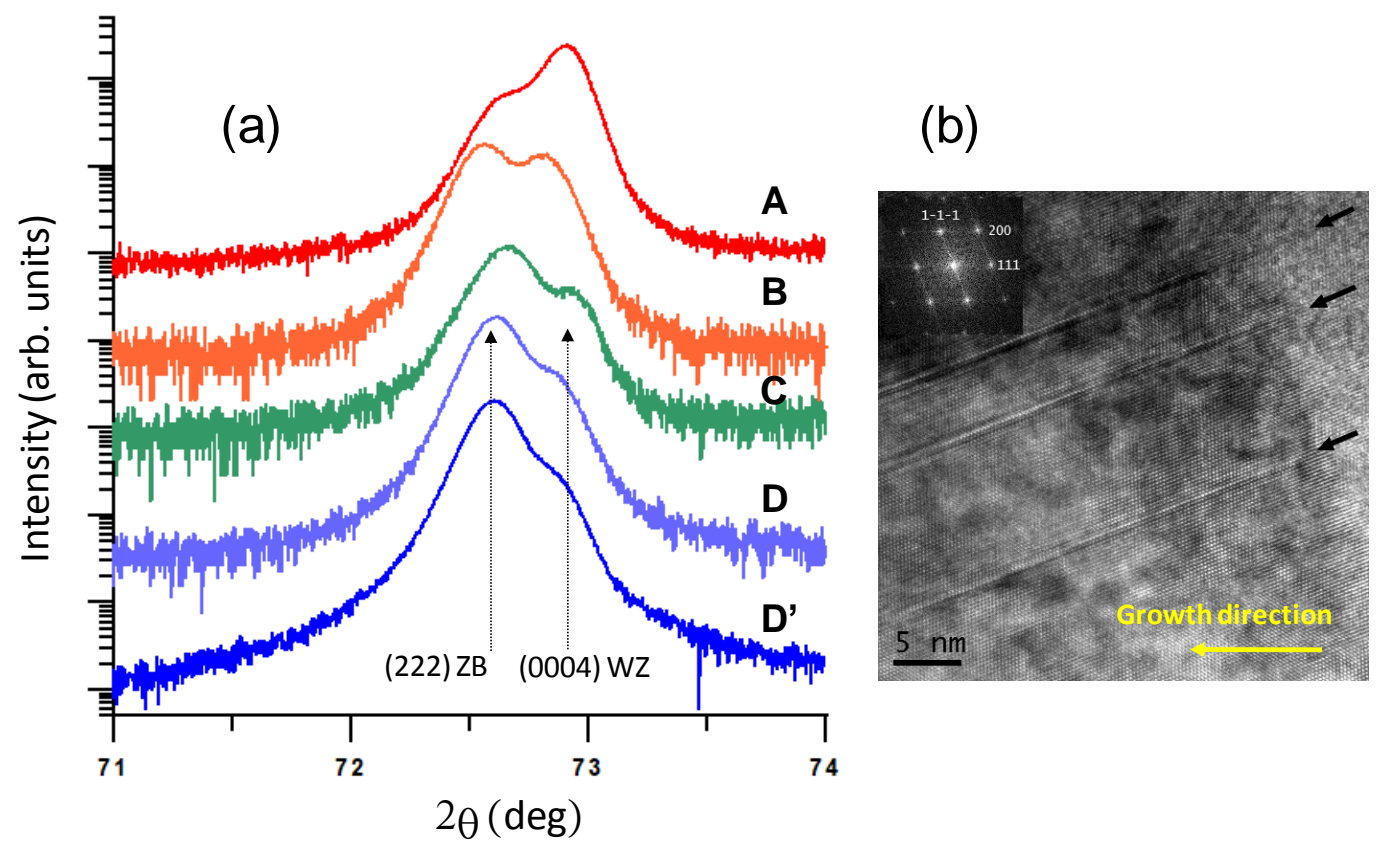

Figure 2: (a) Out-of-plane $2 \theta-\theta$ scans of samples A-D, D' around the WZ (0004) and the $Z B$ (222) reflection of the GaN layers (b) HR-TEM image of sample D' prepared in cross section. The Fourier transform of the image is given in inset and can be indexed in cubic symmetry. A few defects indicated by black arrows correspond to microtwins.

In-plane radial and axial X-ray diffraction scans (using $0.5^{\circ}$ collimators) were performed on sample A along the (0h0) mica direction in order to check the epitaxial relationships between $\mathrm{GaN}$ and mica (see figure 3). It is concluded that the GaN [11-20] direction is parallel to the [010] mica direction. Unfortunately, it was not possible to measure the in-plane epitaxial relation of the ZB phase to mica as the width of the peaks do not allow to resolve the two phases. As concerns WZ phase, these results are consistent with those of Matsuki et al who have speculated that a weak VdW interaction between GaN and mica 
indeed results in an epitaxial relationship governed by the coincidence relationship between both crystalline lattices [10]. This is also consistent with the schematics of figure $3 \mathrm{~b}$, emphasizing a coincidence relationship between $\mathrm{GaN}$ (green) and mica (red) in-plane reciprocal lattices. Interestingly, a single epitaxial relationship of the basal GaN plane was observed with respect to mica, in agreement with the $\phi$ scan data on GaN (11.0) and mica (060) reflections shown in figure 3c.

(a)
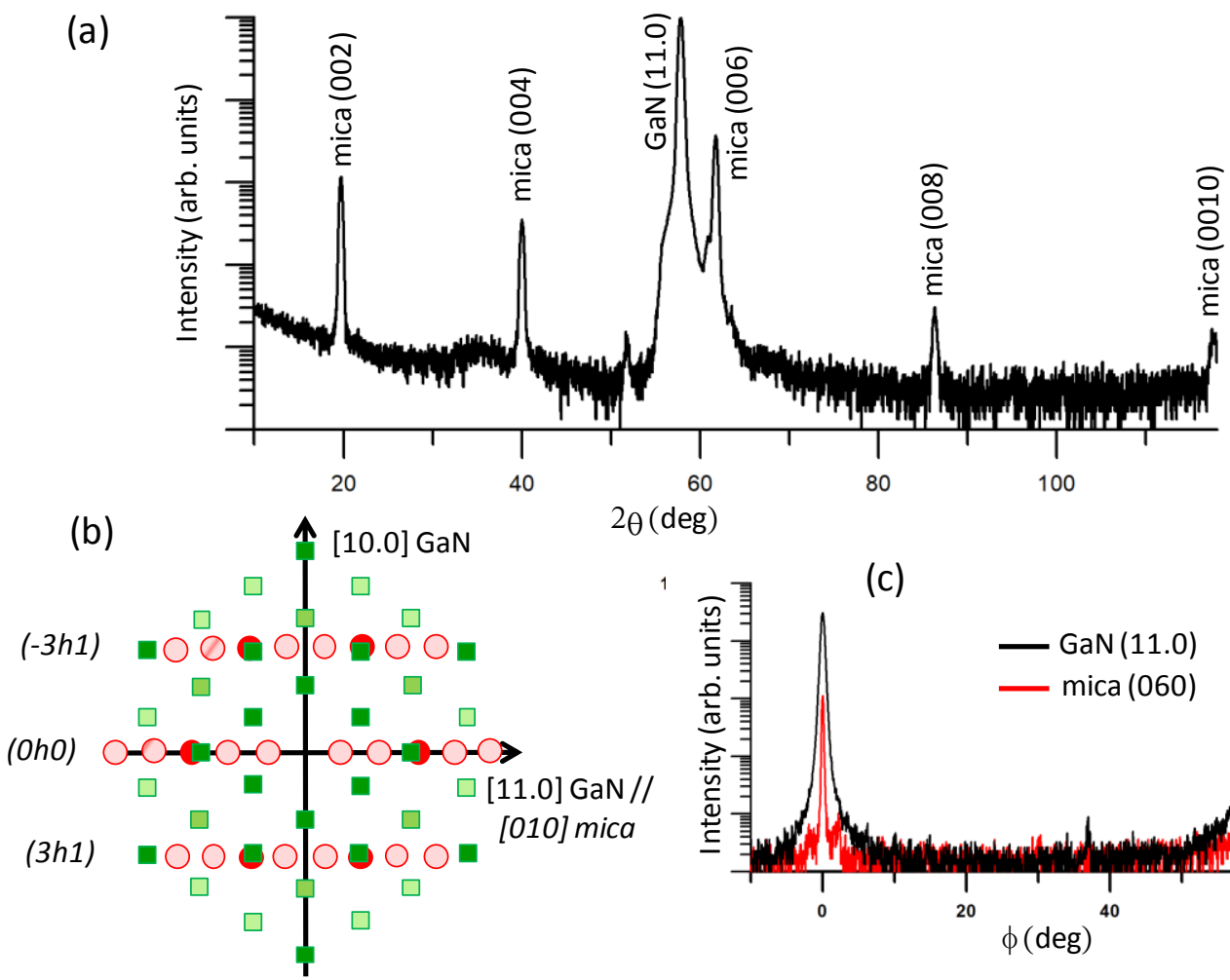

$$
\theta(\mathrm{deg})
$$

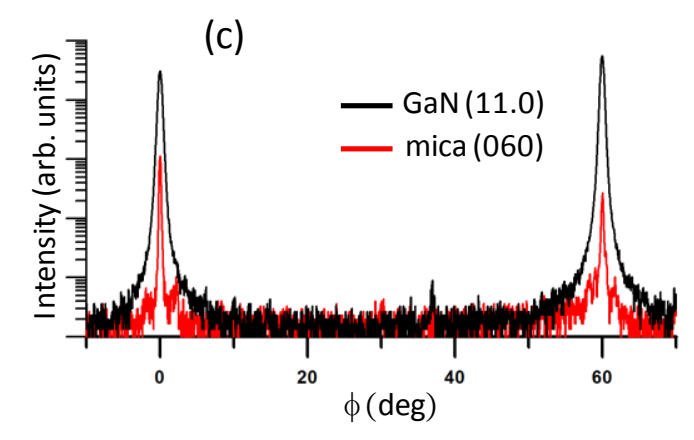

Figure 3: (a) In-plane) $2 \theta-\theta$ scans of sample A. Radial scan along the (Oh0) direction of mica. (b) Schematical superposition of GaN (green squares) and mica (red dots) in-plane reciprocal lattice. The color intensity of the points is a signature of the intensity of the reflection. (c) $\phi$ scan along the (060) reflection of mica and along the (11.0) reflection of the GaN layer.

At this stage, the unique epitaxial relationship between $\mathrm{GaN}$ and mica as well as the demonstration that almost fully $\mathrm{ZB}$ layers can be grown emphasize the importance of nucleation conditions and the growth technique itself, which is suggested by the differences between the present results and those of Matsuki et al obtained using pulse laser deposition [10]. The GaN layers grown using that technique exhibited $\mathrm{WZ}$ structure, $\mathrm{ZB}$ phase being scarcely observed. The difference may come from the residual $\mathrm{N}$ ions present in the plasma in the case of PA-MBE used in the present work, which may damage the substrate. As 
demonstrated in the case of graphene, exposure to $\mathrm{N}$ plasma produces electronic and structural defects [23]. As the mica surface is terminated by a layer of $\mathrm{Si} / \mathrm{Al}$ atoms tetrahedrally coordinated to $\mathrm{O}$, and due to high chemical reactivity of $\mathrm{N}$ with $\mathrm{Si}$, it is expected that $\mathrm{N}$ ions from the plasma can react with the surface.

On the other hand, the formation of phone cord buckles in the layer also suggests that $\mathrm{GaN}$ may be weakly bound to the subjacent substrate, which raises the need to identify more precisely both growth and strain relaxation mechanisms. For this purpose, the GaN layer was peeled off using adhesive tape. XRD experiments in grazing incidence revealed the presence of mica on the backside of the peeled layer, as a further clue of a strong chemical bonding between GaN and mica. Accordingly, it appears that the phone cord buckles observed in figure 1 correspond to a delamination of mica itself, as a clue that the hypothesis of a van der Waals epitaxy growth mode of GaN on mica might be tentatively discarded, at least in the case of plasma-assisted molecular beam epitaxy. The reduced thickness of GaN layers, around $250 \mathrm{~nm}$, combined with their high degree of strain relaxation additionally suggests that only one or a few layers of mica are strongly bound to $\mathrm{GaN}$, with no sizable effect on its final strain state. However, it cannot be excluded at this stage that a $\mathrm{GaN} /$ mica van der Waals interaction stronger than the interaction between mica layers could favour the preferred mica delamination during the peeling-off process.

In order to focus on optical properties at the nanoscale, cathodoluminescence (CL) mapping of samples C and D were performed using an FEI Inspect F50 Field Emission Scanning Electron Microscope (FESEM) operating at $10 \mathrm{keV}$ and $44 \mathrm{pA}$ for the electron beam energy and current, respectively. SEM and panchromatic images (with a spectral width of $20 \mathrm{~nm}$ ) were acquired simultaneously using an in-house CL set-up for the light collection in the FESEM chamber and its focalization at the entrance slit of a Horiba Jobin-Yvon iHR550 spectrometer fitted with an UV enhanced Andor Newton 940 CCD. Importantly, the $10 \mathrm{keV}$ electron penetration depth, around $300 \mathrm{~nm}$, ensures probing the whole of the GaN layers, typically $250 \mathrm{~nm}$ thick, down to the mica substrate. 

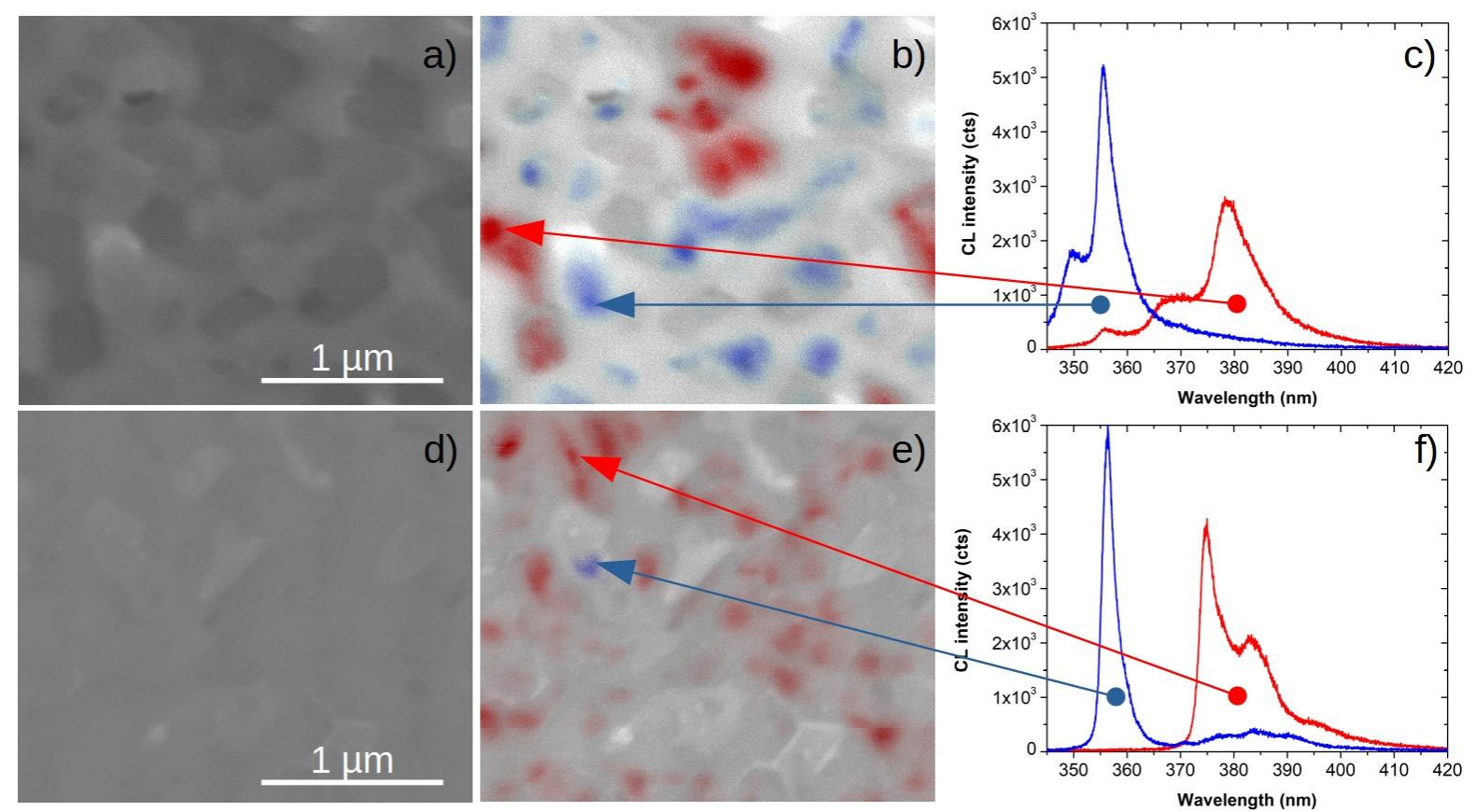

Figure 4: (a), (b), (c): SEM image, SEM image superimposed with bichromatic CL maps at $357 \mathrm{~nm}$ (WZ band edge in blue) and $382 \mathrm{~nm}$ (ZB band edge in red) and CL spectra at spot position indicated by arrow of sample $C$ and $(d),(e),(f)$ of sample $D$.

As shown in figure $4 \mathrm{~b}$, sample $\mathrm{C}$ exhibits a mixture of grains preferentially emitting either at 357 or $382 \mathrm{~nm}$, i.e. either mostly of $\mathrm{WZ}$ or ZB structure, respectively. However, the spectra recorded in the $325-420 \mathrm{~nm}$ range corresponding to two different positions in the sample indicated by the arrows and plotted in figure $4 \mathrm{c}$ reveal a significant overlap of both contributions, suggesting that the phase mixing is not only from grain to grain but is likely present also along the growth axis.

By contrast, the CL mapping of sample $\mathrm{D}$ in figure $4 \mathrm{e}$ reveals that it mostly consists of grains with the ZB structure while grains with the $\mathrm{WZ}$ one are scarcely observed. As shown in figure 4f, no overlap between $\mathrm{WZ}$ and $\mathrm{ZB}$ band edge contributions is present in this case, suggesting a grain to grain phase mixing. This further indicates that each individual ZB (WZ) grain is free of $\mathrm{WZ}(\mathrm{ZB})$ contribution along the growth axis, as a signature that both phases can nucleate directly on the mica surface.

In order to investigate more deeply the relationship between phase mixing and sample morphology, additional cross section CL mapping experiments were performed on sample (D') grown in conditions comparable to those of sample D. The GaN layer was peeled off 
using adhesive tape for the observation. Cross section SEM images superimposed to bichromatic CL maps are shown in figure 5, confirming that ZB phase directly nucleates on the mica substrate, in agreement with a grain-to-grain phase mixing mechanism. Interestingly, spectra corresponding to adjacent WZ/ZB grains (corresponding to points $\mathrm{c}$ and $\mathrm{d}$, respectively) allow one to conclude that $\mathrm{WZ}$ grains are slightly higher than their $\mathrm{ZB}$ counterpart.
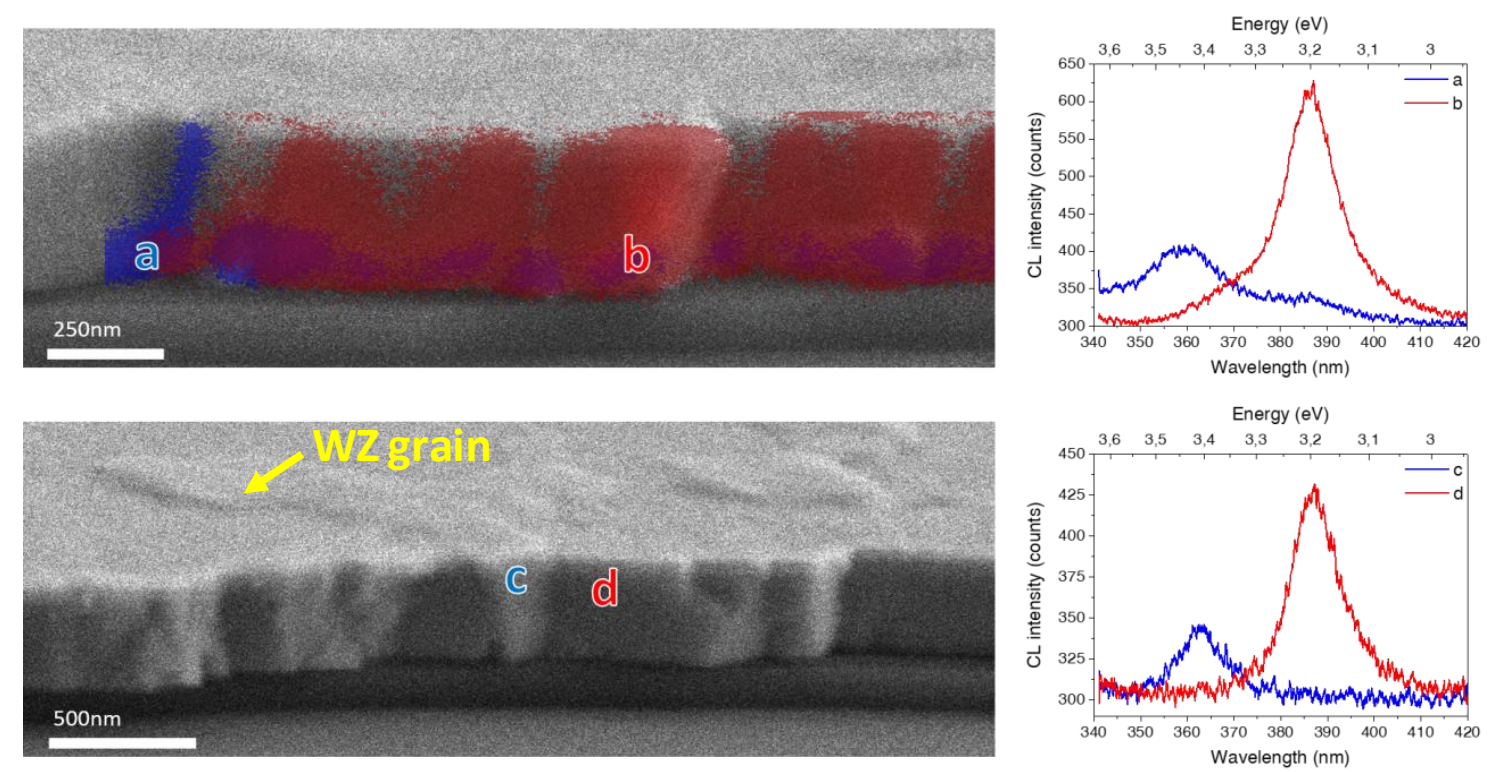

Figure 5: Cross section SEM images superimposed to bichromatic CL maps at $357 \mathrm{~nm}$ (WZ band edge in blue) and $382 \mathrm{~nm}$ (ZB band edge in red) and CL spectra at spot positions indicated in SEM images (sample D').

The polarity of GaN, i.e. the orientation of the Ga-N bond with respect to the normal to the surface, is a fundamental property directly related to the first stages of the nucleation on the substrate. In the case of $\mathrm{WZ} \mathrm{GaN}$, considering the $\mathrm{Ga}-\mathrm{N}$ bond along the c-axis, the vector starting from $\mathrm{Ga}$ and pointing towards $\mathrm{N}$ is conventionally labelled [0001] and corresponds to Ga polarity, i.e. to a Ga-face terminated WZ GaN crystal[24]. By contrast, an anti-parallel orientation of the normal with respect to the Ga-N bond vector is labelled [000-1] and corresponds to $\mathrm{N}$-face termination. As $\mathrm{ZB}$ is only differing from $\mathrm{WZ}$ by the plane stacking sequence, the polarity definition of [0001] oriented $\mathrm{WZ} \mathrm{GaN} \mathrm{and} \mathrm{its} \mathrm{associated} \mathrm{chemical}$ terminating surface also holds for [111] ZB variant. More precisely, in the case of ZB GaN, all the Ga-N bonds have the same orientation and define a given $\langle 111\rangle \boldsymbol{G a N}$ bond vector that 
is used to define the polarity. If the (111) outgoing surface normal $\mathbf{n}$ is positively parallel to the [111] $\boldsymbol{G a N}$ bond vector, the polarity is said to be $\mathrm{Ga}$ and the creation of a (111) surface will tend to break these [111] $\boldsymbol{G a N}$ bonds, leading to a Ga-terminated surface as illustrated in figure $6 \mathrm{a}$. Because the nature of the terminating surface, namely $\mathrm{Ga}$ or $\mathrm{N}$, is affecting the incorporation of impurities during the growth [25] and consequently the properties of engineered electron gases in $\mathrm{GaN} / \mathrm{AlGaN}$ heterostructures [26] the polarity determination is desirable in order to optimize the growth process and the structure of devices.

There is no a priori reason favouring one or the other polarity when growing GaN on mica. Then, in order to clarify this issue, the polarity of the samples was analysed by transmission electron microscopy using the convergent beam electron diffraction (CBED) technique. CBED patterns were acquired with a ThermoFisher Scientific TITAN Ultimate microscope working at $200 \mathrm{kV}$ in microprobe Scanning mode. This mode allows using a relatively large probe of about $2 \mathrm{~nm}$ in diameter with a rather moderate convergent angle of about $0.5 \mathrm{mrad}$ that can be scanned on the sample, providing both an overview of the sample and local diffraction patterns shown in figure 6. Diffraction patterns of ZB GaN were acquired along [11-2] and [1-10], but figure 6 only presents the [11-2] direction for simplicity. In order to determine the polarity one has to determine the relative orientations firstly between the CBED pattern and the GaN structure and secondly between the CBED pattern and the outgoing surface normal $\mathrm{n}$. The first one was determined by simulating CBED patterns at different thicknesses using the jems software[27]. The second one was determined by acquiring an out-of-focus CBED pattern obtained by lowering the sample under the convergent beam (figure 6d). In all the analysed areas N-polarity was observed.
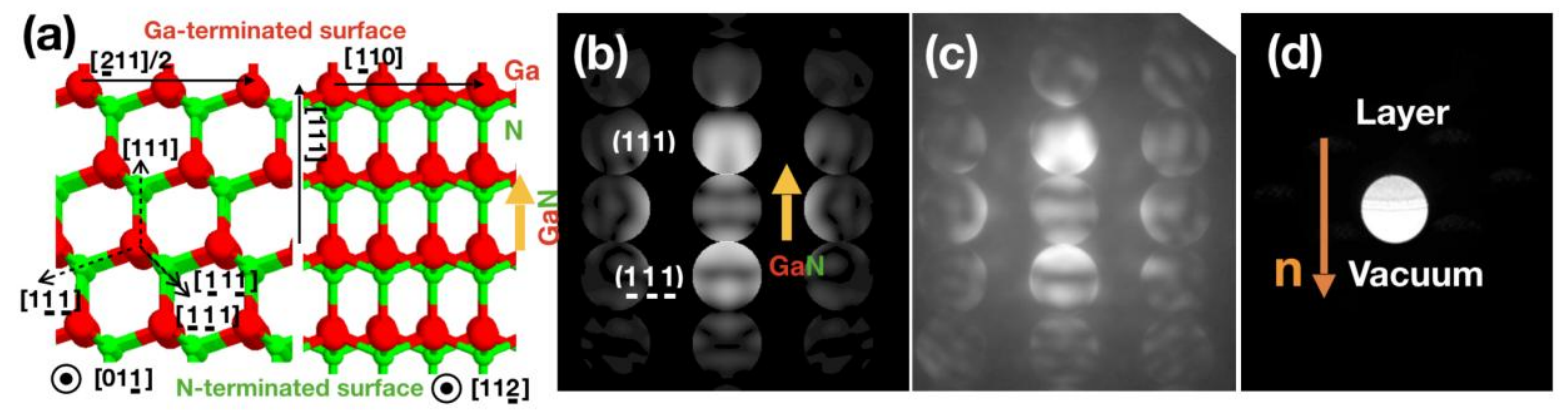

Figure 6: (a) Schematics of the ZB GaN structure observed respectively along the [011] and [11-2] directions recalling the polarity definition for a (111) GaN surface. In our conventions, the [111], [1-1-1], [-11-1] and [-1-11] directions in dotted arrows are the orientations of the GaN bonds. If the [111] GaN bond vector is positively parallel to the 
outgoing surface normal $\boldsymbol{n}$, the layer is said to be Ga-polar; if $\mathbf{G a N}$ and $\boldsymbol{n}$ are antiparallel, which will be the case in Fig 6.c and 6.d, the layer exhibits a N-polarity. (b) indexed [11-2] simulated CBED pattern of a ZB GaN crystal having a thickness of $46 \mathrm{~nm}$. The (-1-1-1) disk, antiparallel to [111], contains dark lines looking like a $\pi$. (c) Experimental CBED pattern $(d)$ Experimental out-focus CBED pattern obtained by lowering the sample: in the direct disk the image of the sample is appearing giving the relative orientation between the surface normal and the CBED.
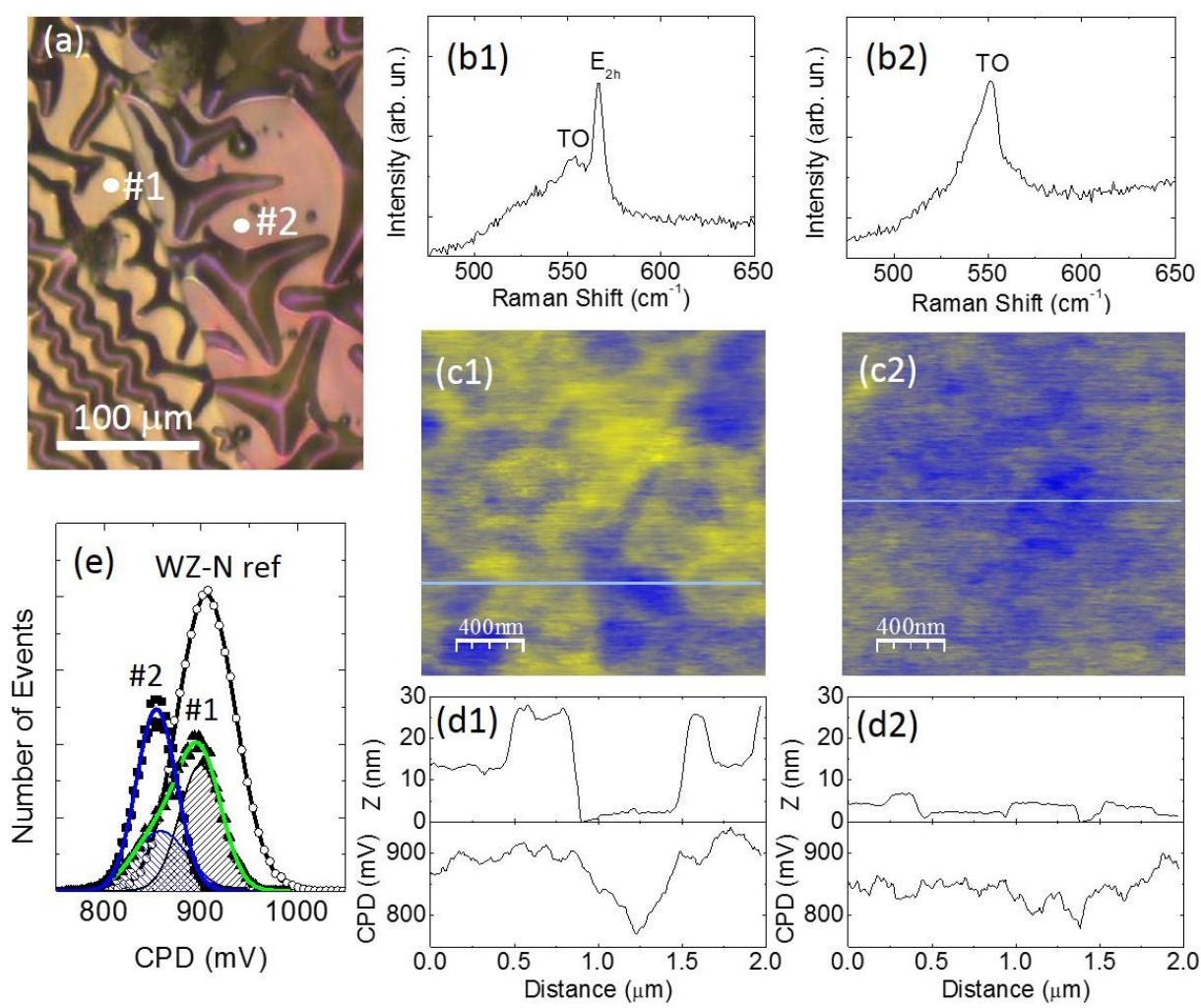

Figure 7: (a) Optical micrograph of sample C. The white dots indicate the points (regions \#1 and \#2) studied with Raman, AFM and KPFM. Region \#1: Raman spectrum (b1), CPD image (color scale spans from 650 (blue) to 1100 (yellow) $\mathrm{mV}$ ) (c1) and topography (top) and CPD (bottom) profile along the blue line (d1). Similar data are given in (b2), (c2) and (d2) for region \#2. (e) Statistical analysis of CPD images (c1) and (c2). The black line with empty dots corresponds to a WZ N polar GaN reference sample.

Complementary to CBED experiments, the polarity of $\mathrm{WZ}$ and $\mathrm{ZB}$ GaN grains in mixed layers was investigated by means of Kelvin probe force microscopy (KPFM) measurements that allow one to probe larger areas of the layers. These KPFM measurements 
were performed with a Nanotec AFM instrument, following a similar procedure as in ref. [28], that is, by using the force gradient as the input signal for the determination of the contact potential difference (CPD) between tip and sample. Pt-Cr coated silicon tips with a nominal force constant of $3 \mathrm{~N} / \mathrm{m}$ were used. Since the mica substrate is insulating, for these measurements the top surface of the samples was grounded. In order to probe the material polarity we have studied sample $\mathrm{C}$ which, as indicated previously, shows regions with different relative $\mathrm{ZB} / \mathrm{WZ}$ phases, depending on location. We have taken advantage of this fact and localized two nearby regions in the sample with different ZB/WZ content. An optical micrograph of the region chosen for the analysis is shown in figure 7a. The white points labeled \#1 and \#2 indicate the locations where Raman and KPFM measurements were performed. The corresponding Raman spectra are shown in figures $7 \mathrm{~b} 1$ and $7 \mathrm{~b} 2$. In \#1 both $\mathrm{WZ}$ and ZB peaks are present in the spectrum, while in $\# 2$ the $\mathrm{WZ}$ peak is absent, indicating pure ZB phase. Subsequently, the same regions were investigated by means of simultaneous AFM and KPFM measurements. From the topography images (supplementary S1) we find that the surface of region \#2 is much flatter than that of region \#1 (RMS $4 \mathrm{~nm}$ versus $12 \mathrm{~nm}$, respectively). The corresponding KPFM images are shown in figures $7 \mathrm{c} 1$ and $7 \mathrm{c} 2$. The CPD of region \#2 is very homogeneous, with a mean value of $854 \mathrm{mV}$. Meanwhile, changes are more notable in region \#1, with variations in CPD of up to $150 \mathrm{mV}$, larger values related with higher grains. These changes are more noticeable in the profiles shown in figures $7 \mathrm{~d} 1$ and $7 \mathrm{~d} 2$, where topography and CPD values can be compared, as well as in figure S1(b) of the supplementary information, where CPD values are superposed to the topographic features displayed with a three dimensional perspective. The statistical analysis of the CPD images is shown in figure 7e. As consequence of its homogeneity, the CPD values of region \#2 give a symmetric Gaussian peak at $854 \mathrm{mV}$. On the other hand, the CPD values of region \#1 are characterized by an asymmetric peak that can be fitted to two Gaussians, with centers at 858 $\mathrm{mV}$ and $900 \mathrm{mV}$. These values roughly correspond to the blue and yellow areas of figure $7 \mathrm{c}$, respectively. From the analysis it is clear that the lower CPD values correspond to ZB, while the higher ones relate to $\mathrm{WZ} \mathrm{GaN}$. A reference GaN sample grown by HVPE (provided by Lumilog) measured with the same kind of tip on its N-polar face gave a mean CPD value of $905 \mathrm{mV}$. Consequently, we conclude that WZ GaN growth on mica takes place along the [000-1] direction. Assuming that the mica/GaN interfacial chemistry does not depend on the plane stacking sequence determining the GaN polytype, it is also concluded that the $858 \mathrm{mV}$ CPD value determined for ZB GaN corresponds to the N-face (111) surface, consistent with 
CBED measurements. In good agreement with CL results, higher regions correspond to WZ $\mathrm{GaN}$, while lower ones have $\mathrm{ZB}$ structure.

\section{Conclusion}

In summary, it was shown that GaN could be grown on mica preserving a single epitaxial relationship, in spite of the large in-plane symmetry mismatch. On the one hand, the single epitaxial relationship points towards the formation of chemical bonding at the interface, possibly related to defects caused by the $\mathrm{N}$ plasma. On the other hand, the observation of buckling and the strain-relaxed state of the GaN layers pleads for delamination of the upper layers of the mica substrate itself. This suggests that the growth mode of GaN on mica is not of van der Waals type although it is also possible that a stronger GaN/mica van der Waals interaction compared to the interaction between mica sheets preferentially favours mica delamination. The growth of mostly ZB GaN layers at high temperature and Ga-rich growth conditions was demonstrated despite the thermodynamically less stable character of the ZB phase with respect to its $\mathrm{WZ}$ counterpart. These features point towards a paradigm change when using mica as a substrate, suggesting a peculiar nucleation mechanism favouring the prevalence of the $\mathrm{ZB}$ phase. Noticeably, the $\mathrm{ZB} / \mathrm{WZ}$ phase ratio was found to increase for increasing temperature. This is particularly remarkable for samples $\mathrm{D}$ and $\mathrm{D}^{\prime}$ grown at $850^{\circ} \mathrm{C}$ and found to consist of almost single ZB phase exhibiting N-polarity. This confirms that crystallographic phase selection is indeed related to the nucleation process itself. As a further clue, CL mapping data suggest that when ZB phase dominates, the GaN layer grown on muscovite mica is formed of separated grains, free of phase mixing along the growth axis. This leads to the conclusion that ZB phase is nucleating directly on mica and that this nucleation process is favoured at high temperature. However, once initiated the growth of ZB $\mathrm{GaN}$, it furthermore appears suitable to use Ga-rich conditions to maintain the $\mathrm{ZB}$ phase purity. Combined to the possibility of easily separating the GaN layer from the mica substrate, these features open the path to the realization of devices taking advantage of the specific properties of $\mathrm{ZB}$ phase.

\section{Acknowlegments}

We thank Dr. B. Beaumont, from Lumilog Company, for graciously providing the free standing GaN sample used for reference in KPFM measurements. A.C. and N.G. acknowledge financial support from Project No. ENE2016-79282-C5-3-R from the Spanish 
MICINN and PROMETEO/2018/123 from Generalitat Valenciana and the expert assistance of SCSIE technical staff. 


\section{References}

[1] Koma A 1992 Van der Waals epitaxy — a new epitaxial growth method for a highly lattice-mismatched system Thin Solid Films 216 72-6

[2] Ueno K, Saiki K, Shimada T and Koma A 1990 Epitaxial growth of transition metal dichalcogenides on cleaved faces of mica J. Vac. Sci. Technol. A Vacuum, Surfaces, Film. 8 68-72

[3] Lin Y C, Ghosh R K, Addou R, Lu N, Eichfeld S M, Zhu H, Li M Y, Peng X, Kim M J, Li L J, Wallace R M, Datta S and Robinson J A 2015 Atomically thin resonant tunnel diodes built from synthetic van der Waals heterostructures Nat. Commun. 6 1-6

[4] Kim J, Bayram C, Park H, Cheng C W, Dimitrakopoulos C, Ott J A, Reuter K B, Bedell S W and Sadana D K 2014 Principle of direct van der Waals epitaxy of singlecrystalline films on epitaxial graphene Nat. Commun. 5

[5] Li Y, Zhao Y, Wei T, Liu Z, Duan R, Wang Y, Xiang Z, Wu Q, Yan J, Yi X, Yuan G, Junxi W and Li J 2017 Van der Waals epitaxy of GaN-based light-emitting diodes on wet-transferred multilayer graphene film Jpn. J. Appl. Phys. 56085506

[6] Liu Y, Xu Y, Cao B, Li Z, Zhao E, Yang S, Wang C, Wang J and Xu K 2019 Transferable GaN Films on Graphene/SiC by van der Waals Epitaxy for Flexible Devices Phys. status solidi 2161801027

[7] Journot T, Okuno H, Mollard N, Michon A, Dagher R, Gergaud P, Dijon J, Kolobov A V and Hyot B 2019 Remote epitaxy using graphene enables growth of stress-free GaN. Nanotechnology 30505603

[8] Gruart M, Feldberg N, Gayral B, Bougerol C, Pouget S, Bellet-Amalric E, Garro N, Cros A, Okuno H and Daudin B 2020 Impact of kinetics on the growth of GaN on graphene by plasma-assisted molecular beam epitaxy Nanotechnology 31115602

[9] Saidi W A 2014 Van der Waals Epitaxial Growth of Transition Metal Dichalcogenides on Pristine and N-Doped Graphene

[10] Matsuki N, Kim T W, Ohta J and Fujioka H 2005 Heteroepitaxial growth of gallium nitride on muscovite mica plates by pulsed laser deposition Solid State Commun. 136 $338-41$ 
[11] Okumura H, Ohta K, Feuillet G, Balakrishnan K, Chichibu S, Hamaguchi H, Hacke P and Yoshida S 1997 Growth and characterization of cubic GaN J. Cryst. Growth 178 $113-33$

[12] As D J, Schikora D and Lischka K 2003 Molecular beam epitaxy of cubic III-nitrides on GaAs substrates Phys. status solidi 0 1607-26

[13] Schörmann J, Potthast S, As D J and Lischka K 2007 In situ growth regime characterization of cubic GaN using reflection high energy electron diffraction Appl. Phys. Lett. 90041918

[14] As D J, Frey T, Schikora D, Lischka K, Cimalla V, Pezoldt J, Goldhahn R, Kaiser S and Gebhardt W 2000 Cubic GaN epilayers grown by molecular beam epitaxy on thin $\beta-\mathrm{SiC} / \mathrm{Si}$ (001) substrates Appl. Phys. Lett. 76 1686-8

[15] Martinez-Guerrero E, Bellet-Amalric E, Martinet L, Feuillet G, Daudin B, Mariette H, Holliger P, Dubois C, Bru-Chevallier C, Nze P A, Chassagne T, Ferro G and Monteil Y 2002 Structural properties of undoped and doped cubic GaN grown on $\mathrm{SiC}(001) \mathrm{J}$. Appl. Phys. 91 4983-7

[16] Daudin B, Feuillet G, Hübner J, Samson Y, Widmann F, Philippe A, Bru-Chevallier C, Guillot G, Bustarret E, Bentoumi G and Deneuville A 1998 How to grow cubic GaN with low hexagonal phase content on (001) SiC by molecular beam epitaxy J. Appl. Phys. 84 2295-300

[17] Cui Y, Lazorov V K, Goetz M M, Liu H, Robertson D P, Gajdardziska-Josifovska M and Li L 2003 Cubic GaN formation in $\mathrm{Mn} / \mathrm{GaN}$ multilayer films grown on $6 \mathrm{H}$ SiC(0001) Appl. Phys. Lett. 82 4666-8

[18] Moon M W, Jensen H M, Hutchinson J W, Oh K H and Evans A G 2002 The characterization of telephone cord buckling of compressed thin films on substrates $J$. Mech. Phys. Solids 50 2355-77

[19] Chen X 2013 Mechanical self-assembly: Science and applications Mech. SelfAssembly Sci. Appl. 9781461445 1-206

[20] Ni Y, Yu S, Jiang H and He L 2017 The shape of telephone cord blisters Nat. Commun. 8 1-6 
[21] Davydov V Y, Averkiev N S, Goncharuk I N, Nelson D K, Nikitina I P, Polkovnikov A S, Smirnov A N, Jacobson M A and Semchinova O K 1997 Raman and photoluminescence studies of biaxial strain in GaN epitaxial layers grown on $6 \mathrm{H}-\mathrm{SiC} J$. Appl. Phys. 82 5097-102

[22] Wei C H, Xie Z Y, Li L Y, Yu Q M and Edgar J H 2000 MOCVD growth of cubic GaN on 3C-SiC deposited on Si(100) substrates J. Electron. Mater. 29 317-21

[23] Feldberg N, Klymov O, Garro N, Cros A, Mollard N, Okuno H, Gruart M and Daudin B 2019 Spontaneous intercalation of Ga and in bilayers during plasma-assisted molecular beam epitaxy growth of $\mathrm{GaN}$ on graphene on $\mathrm{SiC}$ Nanotechnology $\mathbf{3 0}$

[24] Rouviere J L, Weyher J L, Seelmann-Eggebert M and Porowski S 1998 Polarity determination for GaN films grown on (0001) sapphire and high-pressure-grown GaN single crystals Appl. Phys. Lett. 73 668-70

[25] Sumiya M, Yoshimura K, Ohtsuka K and Fuke S 2000 Dependence of impurity incorporation on the polar direction of GaN film growth Appl. Phys. Lett. 76 2098-100

[26] Ambacher O, Foutz B, Smart J, Shealy J R, Weimann N G, Chu K, Murphy M, Sierakowski A J, Schaff W J, Eastman L F, Dimitrov R, Mitchell A and Stutzmann M 2000 Two dimensional electron gases induced by spontaneous and piezoelectric polarization in undoped and doped $\mathrm{AlGaN} / \mathrm{GaN}$ heterostructures $J$. Appl. Phys. 87 33444

[27] JEMS http://www.jems-swiss.ch

[28] Minj A, Cros A, Garro N, Colchero J, Auzelle T and Daudin B 2015 Assessment of Polarity in GaN Self-Assembled Nanowires by Electrical Force Microscopy Nano Lett. $156770-6$ 